\title{
Emergence of A Novel Disease Caused by Staphylococcus Pseudintermedius in Raccoon Dogs (Nyctereutes procyonoides)
}

\author{
Yisong Zhu ${ }^{1}$, Hongmei $\mathrm{Li}^{1}$, Kaiyan $\mathrm{Guo}^{1}$, ziqiang cheng ${ }^{1}$, Faxiao Liu ${ }^{1}$, Yuqing Zhao, \\ Huijun $\mathrm{Guo}^{1}$, and Jianhua Qiu ${ }^{1}$ \\ ${ }^{1}$ Shandong Agricultural University
}

April 28, 2020

\begin{abstract}
This study reports outbreak of a new disease caused by Staphylococcus Pseudintermedius in Raccoon dogs. The disease occurred in a breeding farm of raccoon dogs in Guan County of Shandong Province in China in August of 2019. 47\% (425/896) of raccoon dogs showed the abnormal symptoms; $17.6 \%(75 / 425)$ of them had sever skin and soft tissue infections(SSTIs), dyspnea and sever pathological lesions in lungs, hearts, livers, kidneys, etc; $4.2 \%(18 / 425)$ were dead in 4 weeks. The pathogen of the disease was identified as Staphylococcus pseudintermedius by mass spectrometer detection, microscopic examination and biochemical reaction test. Its nucleotide homology of 16 SrDNA was $100 \%$ with thats of other published strains and its genotype was between American strain and Brazilian strain from other animal species. The isolated Staphylococcus pseudointermediate strain from the diseased raccoon dogs could cause ulceration and suppuration in the skins and sever pathological lesions in some organs of the infected mice, and it is confirmed as a methicillin-resistant Staphylococcus pseudintermedius (MRSP) strain by the amplification of mecA gene; and 12 sensitive drugs were screened by drug sensitivity test. Full attention should be paid to the great economic loss and the potential zoonotic risk caused by the Staphylococcus pseudintermedius in raccoon dogs, and this study can provide a reference for the clinical diagnosis and treatment of this new disease.
\end{abstract}

\section{Emergence of A Novel Disease Caused by Staphylococcus Pseudintermedius in Raccoon Dogs (Nyctereutes procyonoides)}

Yisong Zhu" , Hongmei Li ${ }^{\#}$, Kaiyan Guo , Ziqiang Cheng, Faxiao Liu, Yuqing Zhao, Huijun Guo*, Jianhua Qiu *

Provincial Key Laboratory of Animal Biotechnology and Disease Control and Prevention; College of Animal Science and Veterinary Medicine, Agricultural University, Tai'an, 271018, .

Abstract This study reports outbreak of a new disease caused byStaphylococcus Pseudintermedius in Raccoon dogs. The disease occurred in a breeding farm of raccoon dogs in Guan County of Shandong Province in in August of 2019. 47\% (425/896) of raccoon dogs showed the abnormal symptoms; $17.6 \%(75 / 425)$ of them had sever skin and soft tissue infections(SSTIs), dyspnea and sever pathological lesions in lungs, hearts, livers, kidneys, etc; $4.2 \%$ (18/425) were dead in 4 weeks. The pathogen of the disease was identified asStaphylococcus pseudintermedius by mass spectrometer detection, microscopic examination and biochemical reaction test. Its nucleotide homology of $16 \mathrm{SrDNA}$ was $100 \%$ with thats of other published strains and its genotype was between American strain and Brazilian strain from other animal species. The isolated Staphylococcus pseudointermediatestrain from the diseased raccoon dogs could cause ulceration and suppuration in the skins and sever pathological lesions in some organs of the infected mice, and it is confirmed as a methicillin-resistantStaphylococcus pseudintermedius (MRSP) strain by the amplification of mecA gene; and 12 sensitive drugs were screened by drug sensitivity test. Full attention should be paid to the great economic 
loss and the potential zoonotic risk caused by theStaphylococcus pseudintermedius in raccoon dogs, and this study can provide a reference for the clinical diagnosis and treatment of this new disease.

Keywords: Raccoon dog; Staphylococcus pseudintermedius ; Skin and soft tissue infections; Zoonotic pathogen; Methicillin-resistant

\section{Introduction}

Staphylococcus pseudintermedius (S. pseudintermedius) is a gram-positive and facultative anaerobic conditional pathogen and survives in the skin or mucosa of dogs as a common symbiotic bacterial (Bannoehr and Guardabassi, 2012). Due to the decline of the dog's resistance, it can cause severe inflammation, dysfunctions, ulcer in some organs such as skin, external otitis, urinary tract, etc(Windahl et al., 2012; Somayaji et al, 2016). Before 2005, the classification ofS. pseudintermedius had been confused and any non-pigmented hemolytic coagulase positive staphylococci isolated from dogs was often referred to as S. intermedius (Sasaki et al., 2007). In 2005, it was finally defined as S. pseudintermedius by phenotypic characterization and molecular reclassification (Devriese et al., 2005). In the past decade, most cases caused by it occurred in companion dogs (Bannoehr and Guardabassi, 2012; Moodley et al., 2014), and a few in other animals (Iwata et al., 2018), even in human (Van Hoovels et al., 2006; Somayaji et al., 2016a), but few in raccoon dogs.

Raccoon dog is a wild canine animal, and is widely raised as a valuable fur animal all over the world (Ahola et al. 2007). About 20 million raccoon dogs are sold in every year and 29.9\% of them are from (Li et al., 2018). Of course, some famous raccoon dog species such as Local Wusuli raccoon dogs and Red raccoons have been developed in this area and bring remarkable economic benefit to the local farmers(Sun et al., 2012; Ma et al., 2018b). However, with the expansion of breeding scale, diseases caused by pathogenic microbes have become an important potent risk for raccoon dog development and some new diseases accidently occurred (Shao et al., 2014; Feng et al., 2014; Xu et al., 2016; Yu et al, 2018; Nie et al., 2019). This study focuses a new outbreak disease caused by Staphylococcus pseudintermedius in raccoon dogs and can help for its diagnosis, treatment and prevention in clinics.

\section{Materials and methods}

\section{Cases resource, samples collection, experimental mice}

The disease occurred in a breeding farm with about 890 raccoon dogs in Guan County of Shandong Province in August, 2019; and about half of the raccoon dogs were abnormal and several decades of them showed unstable standing, dyspnea, sever swelling in some joints; and a few died for that. Some important information on this disease was recorded. Three raccoon dogs with typical symptom were carefully examined and some tissues samples were collected to be used for identifying the pathogens of this disease.

The special pathogenic free (SPF) BALb/c mice were purchased for artificial-infection-test from Experimental Animal Center of Shandong, of .

\section{Mass spectrometer detection}

Three diseased raccoon dogs' tissues including lung, heart, liver, kidney, pus and lymph node were collected aseptically. Each tissue sample was inoculated into TSA culture medium and cultured for 18-24h at 37. The single colony was picked up to smear on the MALDI target plate and detected using a automatic rapid biomass spectrum detection system (IVD MALDI Biotyper, Bruker Daltonic Inc, U.S.A.) according to the instructions, and then the detected results of the samples were compared with the microbial protein fingerprint in the database and the pathogenic microbes were statistically analyzed.

\section{Bacterial staining and microscopic examination}

According to previously published methods (Devriese et al., 2005; Song et al, 2020), the pathogenic bacterial was cultured in glass dishes with solid medium and the single colony was inoculated aseptically into the clean glass slides, evenly smeared and fixed, then stained with Gram staining reagents, and examined under a microscope for describing the morphological characteristics of the pathogenic bacterial. 


\section{Biochemical reaction test}

According to previously published methods (Devriese et al., 2005; Sasaki et al., 2007), the isolated pathogenic bacterial was inoculated into many micro-biochemical reaction tubes with the culture medium of sucrose, urea, arginine dihydrolase, xylose, lactose, mannose, wood alcohol, maltose, trehalose, mannitol, N-acetyl glucosamine, nitrate, VP-MR, fructose, melibiose, sorbitol, respectively, and then cultured at 37 for $24-36 \mathrm{~h}$. The results of these reactions were recorded and comparatively analyzed with the clinical microbiological diagnosis map for determining the genus of bacteria.

\section{Amplification of $16 \operatorname{SrDNA}$ and $m e c A$ genes}

According to the instruction of DNA extraction kit, the genomic DNA of the isolated bacterial strain was extracted. The primers (Forward:5'-AGAGTTTGATCCTGGCTCAG-3', Reverse:5'-GGTTACCTT GTTACGACTT -3') and the conditions (95 C for $1 \mathrm{~min} ; 30$ cycles of $95 \mathrm{C}$ for $30 \mathrm{~s}, 55 \mathrm{C}$ for $30 \mathrm{~s}$ and $72 \mathrm{C}$ for $90 \mathrm{~s}$ followed by 1 cycle of $72 \mathrm{C}$ for $10 \mathrm{~min}$ ) were used for amplifying $16 \mathrm{SrDNA}$ gene with the size of $1465 \mathrm{bp}$; the primers (forward:5'-GTAGAAATGACTGAACGTCCGATAA-3'; Reverse :5'-CCAATTCCACATTGTTTCGGT CTAA-3') and the conditions (95 C for $1 \mathrm{~min} ; 30$ cycles of $95 \mathrm{C}$ for $30 \mathrm{~s}, 55 \mathrm{C}$ for $30 \mathrm{~s}$ and $72 \mathrm{C}$ for $90 \mathrm{~s}$, followed by 1 cycle of $72 \mathrm{C}$ for $10 \mathrm{~min}$ ) were used for amplifying mecA gene with the size of $310 \mathrm{bp}$. The PCR products were observed by $1 \%$ agarose gel electrophoresis, and then were sequenced by Beijing Liuhe Huada Genome Technology Co., Ltd.

The $16 \operatorname{SrDNA}$ sequence of the isolated bacterial strain was compared with that of the published other strains in GenBank of NCBI, and its homology and phylogenetic tree were analyzed using DNA Star MEGA7 software.

\section{Pathogenicity caused by the isolated pathogenic bacterial in mice}

The isolated pathogenic bacterial strain was inoculated into TSB medium and cultured until the logarithmic growth phase at 37 . The colony-forming units (CFU) of the bacterial was calculated by 10 -fold dilution according to the published method (Savini et al., 2013; Ma et al., 2018a). Six BALb/c mice from S1 to S6 were intraperitoneally inoculated with $1 \times 10^{9} \mathrm{CFU} / \mathrm{mL}$ of bacterial solution, each of which was $0.2 \mathrm{~mL}$. Control mice from $\mathrm{C} 1$ to $\mathrm{C} 2$ were intraperitoneally inoculated with sterilized TSB medium, each with 0.2 $\mathrm{mL}$. After inoculation, the mice were observed for describing their clinical symptoms for two weeks and then were dissected for assessing the pathological lesions in some internal organs. Finally, the pathogenic bacterial in the inoculated mice was isolated and identified according to the previous referred methods.

\section{Drug sensitivity test}

According to the Kirby-Bauer disk method designated by Clinical and Laboratory Standards Institute (CLSI), 24 antimicrobial agents were used for drug sensitivity test. The specific operations were as follows: the isolated bacterial strains were picked up and cultured in TSB medium at 37 for $18 \mathrm{~h}-20 \mathrm{~h}$. The $0.2 \mathrm{~mL}$ bacteria suspension was taken on TSA medium and smeared evenly with a coating rod. After standing for a few minutes, the drug sensitive tablets were spread equidistantly on the surface of the plate with sterile tweezers and cultured at 37 for $24 \mathrm{~h}$. The diameter of bacteriostatic zone was measured and its sensitivity was determined according to CLSI standard.

\section{Data statistical analysis}

The data are represented as the mean + - standard deviation (?X +-SD). The inter-group differences were analyzed using ANOVA software followed by Student-Newman-Keuls tests of multiple comparisons. $P<0.05$ was considered statistically significant.

\section{Results}

The morbidity sketch, clinic symptom and pathological lesions

According to the statement of the raccoon dogs' owners and the scene investigation, the clinic features of the diseased raccoon dogs are described as following: the first case occurred 4 weeks before; in a recent month, 
$47 \%(425 / 896)$ of raccoon dogs showed some abnormal symptoms, and $17.6 \%$ (75/425) among the abnormal ones were too severe to stand or walk, and $4.2 \%(18 / 425)$ were dead. The disease in young raccoon dogs was earlier that that in adult ones, there were no difference between the male and the female.

The symptoms in the early stage: depression, slow responses, loss of appetite, significant reduction of food intake, increase of body temperature by $0.5 \sim 1.0$, disordered coat, curling, and mucus secretion in the nasal cavity.

The symptoms in the typical clinic stage: the disease in most raccoon dogs was characterized by the symptoms such as suppuration of skin or/and joints and severe respiratory dysfunction. The suppurative focus mainly occurred in the limb joints, skins, neck, shoulder, groin, etc; sometimes pustule burst with yellow-white pus, blood and tissue fluid. The respiratory dysfunction showed cough, sneezing, asthma, shortness of breath, cyanosis of visible mucosa, sticky secretion in the nasal cavity, severe purulent nasal juice, dyspnea and abdominal breathing in most sick raccoon dogs.

The symptoms in the later stage: weak physique, emaciation, unstable standing, multiple organs failure, death.

Pathological autopsy: The diseased raccoon dogs in the typical clinic stage were dissected and the lesions were as following: swollen with ulceration (Fig.1A) and yellow-white pus (Fig.1B) in the tarsal joint of the hind limb; some or diffuse hemorrhagic spots or plaques on the surface of the bladder (Fig.1C); lots of effusion fluid in pleural, many hemorrhagic spots in diaphragm (Fig.1D); lots of mucous secretions in the larynx and trachea, and many hemorrhagic spots, hyperemia, white inflammatory exudation in the lungs (Fig.1E); needle-like hemorrhagic spots in the epicardium and no pericardial effusion in the pericardium (Fig.1F); hepatomegaly, lobulation, partial yellowing, gallbladder filling (Fig.1G); clear hemorrhagic spots in the kidney, whitening of renal capsule (Fig.1H); splenomegaly, catarrhal inflammation in intestinal mucosa; congestion and hemorrhagic spots in jejunal mucosa; many bleeding spots in the initial segment of ileum; many bleeding spots in some intestinal lymph nodes.

Based on the above clinic symptoms, the disease in raccoon dogs was suspected to be Staphylococcus infection.

\section{The pathogen of the disease was identified as $S$. pseudintermedius by mass spectrometery}

To obtain the pathogenic microbes, the samples from six tissues of the diseased raccoon dogs were inoculated onto the specific TSA medium in Petri Dishes and the cultured bacterial strains were detected using a mass spectrometer. The results in Table 1 showed that 8 species of bacteria were identified from the pus samples of the suppurative focuses, 5 species from Lymph nodes, 4 species from the lungs and liver, 2 species from the kidney. Among these isolated bacteria strains, two strains including staphylococcus pseudintermedius (S. pseudintermedius) and enterococcus faecalis (E.F) were common and the isolated positive ratios of $S$. pseudintermedius in all the tissues were the highest except in pus. So it was deduced that the pathogen of the disease in raccoon dogs might be $S$. pseudintermedius .

\section{The isolated bacterial strain was a Gram-positivestaphylococcus}

To obtain morphological and staining characteristics of the pathogen, the isolated strain grew into a small colony with a diameter of $1 \sim 2 \mathrm{~mm}$ and showed the specific morphological character with light gray, moist, round, uplifted, smooth and neat edge (Fig. 2A).

Then the single colony was stained with Gram's staining reagent and examined by a microscope. The colony is positive to Gram's staining and showed the morphological character with cocci, single or double clusters, or short-chain and long-chain arrangement under oil microscope (Fig. 2B). These results indicate that the pathogenic bacterial belongs to Staphylococcus.

\section{Staphylococcus pseudintermedius confirmed by biochemical test}

To determine the subgroup of the isolated staphylococcus, its biochemical reactions to 16 designated reagents were tested. The results in Table 2 showed that the designated reagents including sucrose, urea, arginine di- 
hydrolase, xylose, lactose, mannose, maltose, trehalose, N-acetylglucosamine and fructose are positive to the isolated strain, but negative for wood alcohol, mannitol, nitrate, VP-MR, melibiose and sorbitol. Especially, the positive responses to maltose, trehalose and mannitol in Fig.3 can confirm that the isolated pathogenic bacterial isStaphylococcus pseudintermedius, but not Staphylococcus intermedia and Staphylococcus delphini

\section{Genotype of the isolated $S$. pseudintermedius determined by 16SrDNA sequence analysis}

To determine the genotype of the isolated $S$. pseudintermedius , 16SrDNA gene was amplified from the strain samples and its homology and phylogenetic tree were analyzed using DNA Star software. The results in Fig.4 showed that 16SrDNA gene with the size of $1465 \mathrm{bp}$ was successfully amplified, and its nucleotide homology with that of other nine published strains was 100\% (Fig.5); and 16SrDNA gene of the isolated strain was named as $S p-S D A U$. The phylogenetic analysis showed that $S p-S D A U$ strain was between American strain (GenBank:FJ536213) and Brazilian strain (GenBank:KX242544) from other animals (Fig. 6).

\section{Pathogenicity of the isolated S. pseudintermedius in mice}

To assess the pathogenicity of the isolated $S$. pseudintermedius, 6 mice were artificially inoculated using the purified bacterial. After 14 days post inoculation (dpi), the symptoms such as lethargy, depression, loss of appetite, closing eyes, fear of cold, drowsiness, crouching in cages, fur wrinkles occurred in all the six mice; in five of six mice, the obvious ulceration and suppuration appeared in the abdominal skins or hind limb (Fig. 7); but none of six mice was dead in two weeks. After anatomy, swelling and bleeding in varying degrees occurred in the lungs, hearts, livers, kidneys; even hemorrhage and necrosis did in some mice (Fig. 8), which were consistent with those of the diseased raccoon dogs. Correspondingly, the pathogen was isolated from the ulcerative skin and lungs of the infected mice, and was identified as S. pseudintermedius . It was confirmed that the isolated S.pseudointermediate from the diseased raccoon dogs can cause sever lesions in mice.

\section{Amplification of $m e c A$ gene and screening of sensitive drugs}

To analyze the resistance of the isolated bacteria and screen the sensitive drugs against it, the resistant mecA gene was amplified and drug-sensitivity test was conducted. The results in Fig.9 showed that the mecA gene with the size of $310 \mathrm{bp}$ could be detected from the isolated strains using PCR, which suggests that the isolated strain is methicillin-resistant. The results in Table 3 showed that the isolated S.pseudintermedius strain is sensitive to twelve drugs including enrofloxacin, ofloxacin, neomycin, chloramphenicol, meropenem, and amikacin, ciprofloxacin, polymyxin B, streptomycin, cephalothin, florfenicol, erythromycin; but resistant to nine drugs including kanamycin, gentamicin, lincomycin, compound sulfamethoxazole, sulfamethoxazole, tetracycline, aztreonam, penicillin $\mathrm{G}$ and vancomycin; especially, completely resistance to lincomycin, compound sulfamethoxazole and aztreonam.

\section{Discussions}

S. pseudintermedius is a novel Staphylococcus specieidentified in the last decade (Devriese et al., 2005) and this organism has been commonly associated with skin and soft tissue infections (SSTIs) in pet dogs (Chuang et al., 2010; Savini et al., 2013; Stegmann et al., 2010), but few raccoon dogs cases have been described. As an important fur animal, raccoon dog is being widely raised in China and its SSTIs and zoonotic diseases caused by the pathogens are of particular concern (Wang et al., 2014; Xu et al., 2016; Wioletta and Maria, 2018). In this study, about $47 \%$ (425/896) of raccoon dogs were infected by S. pseudintermedius and $17.6 \%$ of them had sever SSTIs and $4.2 \%$ of them were dead in 4 weeks. This is a new threaten to raccoon dogs!

In this study, the clinical symptoms of the raccoon dogs were described according to the characteristics of the disease at different stages and the symptom including suppuration of skin or/and joints and severe respiratory dysfunction was recommended to be used as one of important basises of clinical diagnosis for S. pseudintermedius infection in raccoon dogs. In addition, the pathological lesions in many organs of the diseased raccoon dogs were also examined and described, which greatly enriches the clinical data of the disease. 
It is very difficult to determine the pathogens of the disease in clinic, because SSTIs and respiratory dysfunction can be caused by many pathogens, especially staphylococcus (Rashid and Kravitz, 2015). In the past, this disease in pet dogs had been misdiagnosed as S. intermedius and other bacteria infection (Sasaki et al., 2007; Bannoehr et al., 2007; BöRjesson et al., 2015). The mass spectrometer detection has been recommended as an effective method for diagnosing it (Silva et al., 2015; Somayaji et al., 2016; Wang et al., 2017). According to the results in this study, this method can indeed do that from all the tissues samples, but it is noteworthy that there are 8 species of bacteria identified from the pus tissue on the skin, and some of them might severely interfere the correct judgment on the pathogens; whereas, much less in the visceral organs such as lung and kidney. Therefore, it is better to aseptically collect samples from deep tissues for detecting the pathogens using mass spectrometry.

Usually, three species of S. intermedius group (SIG) including S. pseudintermedius, S. intermedia and $S$. delphini can effect the judgment for the pathogens of SSTIs (Fitzgerald, 2009). To distinguish them, some biochemical reactions to some designated reagents should be tested. According to the previous reports, $S$. pseudintermedius is positive to maltose and trehalose, but negative to mannitol; $S$. intermedia is positive to trehalose and mannitol, but negative to maltose; S. delphini is positive to maltose and mannitol, but negative to trehalose (Bannoehr and Guardabassi, 2012; Sasaki et al., 2007; Devriese et al., 2005). The results of biochemical reactions of the isolated pathogen in this study were exactly the same as previously reported, so we deduced that the isolated pathogen should be $S$. pseudintermedius. This can supply a reference for distinguishing $S$. pseudintermedius infection without mass spectrometer in veterinary clinics.

In some previous reports, S. pseudintermedius has been concerned as a potential zoonotic pathogen (Stegmann et al., 2010; Somayaji et al. 2016b). To evaluate the pathogenicity of the isolated S. pseudintermedius from raccoon dogs on other animals, the mice were inoculated with $1.8 \times 10^{9} \mathrm{CFU}$ of $S$. pseudintermedius and most of them developed skin abscess, ulcer, dyspnea, and sever pathological lesions in some important organs in $14 \mathrm{dpi}$, which were almost identical with those of the diseased raccoon dogs. The results suggest that the isolated $S$. pseudintermediuscan not only cause sever lesions in raccoon dogs, but also in mice. Although $S$. pseudintermediu has been previously reported as a zoonotic pathogen, yet its pathogenicity on cross-species is rarely evaluated. This study can supply some important data for that.

Some studies suggest that $S$. pseudintermedius can be divided into methicillin-sensitive $S$. pseudintermedius (MSSP) and methicillin-resistant S. pseudintermedius (MRSP) (Ruscher et al., 2010; van Duijkeren et al., 2011). Methicillin resistance is mediated by the mecA gene. The mecA gene is located in the mobile genetic element $S C C m e c$, which encodes the penicillin binding protein (PBP) and has a low affinity for $\beta$-lactam antibiotics (Chambers et al., 1997; Bannoehr and Guardabassi, 2012; Ane et al, 2012) When the bacteria were confirmed to contain mecA gene by PCR method, it showed that the bacteria were methicillin-resistant (Chambers, 1997). In recent years, the incidence of MRSP has been increasing, which has brought more and more challenges to clinical treatment against it (Moodley et al., 2014; Mccarthy et al., 2015; Richards et al., 2018). In this study, the resistant gene of $m e c A$ was amplified from the isolated strain, which indicates that it is a MRSP strain, like previous reported other strains.

Interestingly, how many antibiotics are sensitive or resistant to it? The results of drug sensitivity test showed that the strain was highly sensitive to 12 antibiotics and most of them were quinolones and aminoglycosides, and a few of them were chloramphenicol, macrolides and polypeptides, these indicate that the isolated strain has not develop serious multi-drug resistance like the global epidemic MRSP strains, although it belonged to MRSP. In addition, according to the results of drug sensitivity test, the diseased raccoon dogs were treated with some of sensitivity drugs such as quinolones and most of them completely recovered in two weeks, this greatly helps the treatment of this disease in clinical practice.

\section{Conclusions}

This study reports a new disease caused by Staphylococcus pseudintermedius in raccoon dogs and describes its clinical symptoms and pathological lesions in many internal organs; the pathogen of this disease was identified by many methods and belongs to MRRP strain; the isolated $S$. pseudintermedius strain from 
raccoon dogs can cause severe SSTIs and pathological lesions in the infected mice. This is a first report on the disease in raccoon dogs.

\section{Acknowledgements}

This study was funded by the Key Research and Development Program of Shandong Province (Important Science and Technology Innovation Project)(2019JZZY010735) and by Funds of Shandong Province Modern Agricultural Technology System Innovation Team Program (SDAIT-21-10) and by Funds of Shandong "Double Tops" Program.

\section{Conflict of interest}

The authors declare that they have no competing interests.

\section{Esthetic statement}

This experiment and all the animal's materials in this study were approved by the Animal Ethics Committee of Shandong Animal Protection and Welfare Institute (Number: SDAU-2019-040), and all procedures related to the animals and their care conformed to the internationally accepted principles as found in the Guidelines for Keeping Experimental Animals issued by the government.

\section{Data Sharing}

The data that support the findings of this study are available from the corresponding author upon reasonable request

\section{References}

Ahola, L., HäNninen, S. and Mononen, J. (2007). A note on stereotyped behaviour in pair and group-housed farmed juvenile raccoon dogs. Applied Animal Behaviour Science ,107,174-180.

Bannoehr, J. and Guardabassi, L. (2012). Staphylococcus pseudintermedius in the dog: taxonomy, diagnostics, ecology, epidemiology and pathogenicity. Veterinary Dermatology , 23, 253-256.

Bannoehr, J., BenZakour, N.L., Waller, A.S., Guardabassi, Luca., Thoday, K.L., van den Broek A.H.M. and Fitzgerald, J.R. (2007). Population genetic structure of the Staphylococcus intermedius group: insights into agr diversification and the emergence of methicillin-resistant strains. Journal of Bacteriology , 189, 8685-8692.

BőRjesson, S., Gómez-Sanz, E., Ekstrőm, K., Torres, C. and Grönlund, U. (2015). Staphylococcus pseudintermediuscan be misdiagnosed as Staphylococcus aureusin humans with dog bite wounds. European Journal of Clinical Microbiology 6 Infectious Diseases , 34, 839-844.

Chambers, H.F. (1997). Methicillin resistance in staphylococci: molecular and biochemical basis and clinical implications. Clinical Microbiology Reviews , 10, 781-791.

Chuang, C.Y., Yang, Y.L., Hsueh, P.R. and Lee, P.I. (2010). Catheter-related bacteremia caused by staphylococcus pseudintermedius refractory to antibiotic-lock therapy in a hemophilic child with dog exposure. Journal of Clinical Microbiology , 48, 1497-1498.

Devriese, L.A., Vancanneyt, M., Baele, M., Vaneechoutte, M., Graef, E., Snauwaert, C., Cleenwerck, I., Dawyndt, P., Swings, J., Decostere, A, and Haesebrouck, F. (2005). Staphylococcus pseudintermedius sp. nov. a coagulase-positive species from animals. International Journal of Systematic \& Evolutionary Microbiology , 55, 1569-1573.

Feng, H.X., Liu, Y.Y., Song, Q.Q, Ling, Z.S., Zhang, F.X., Zhu, Y.L., Jiang, S.J., Xie, Z.J. (2014). Interspecies transmission of canine influenza virus $\mathrm{H} 5 \mathrm{~N} 2$ to cats and chickens by close contact with experimentally infected dogs. Veterinary Microbiology , 170, 414-417. 
Fitzgerald, J. (2009). The Staphylococcus intermedius group of bacterial pathogens: species reclassification, pathogenesis and the emergence of methicillin resistance. Vet Dermatol ,5-6, 490-495.

Iwata, K., Kasuya, K., Takayama, K., Nakahara, Y., Kobayashi, Y., Kato, A., Senba, H., Yanagisawa, M. and Shibahara, T. (2018). Systemic Staphylococcus pseudintermedius infection in an arctic fox (Vulpes lagopus) with severe multifocal suppurative meningoencephalitis and nephritis. The Journal of veterinary medical science , 80,1219-1222

Li, G.Y., Bao, K., Zhang, X., Si, F.F. and Yan, S. (2018). Review on the Development of Special Economic Animal Breeding Industry in China.Journal of Agriculture , 8, 140-144 (In Chinese).

Ma, L.L., Sun, Z.H., Xu, Y.L., Wang, S.J., Wang, H.N., Zhang, H., Hu, L.P., Sun, X.M., Zhu, L., Shang, H.Q., Zhu, R.L. and Wei, K. (2018a). Screening host proteins required for bacterial adherence after H9N2 virus infection. Veterinary Microbiology, 213, 5-14.

Ma, W.J.,Pang, J.Y.,Wang, K.,Shi, Y.F.,Tang, D.H.,Guo, H.J. and Qiu, J.H. (2018b). Components and Characteristics of Meat /Fat of Artificial Rearing Silver-frost Fox and Wusuli Raccoon Dog. Journal of Economic Animal , 22, 187-192 (In Chinese).

Mccarthy, A.J., Harrison, E.M., Kinga, S.M., Leggett, B., Waller, A., Holmes, M.A., Lloyd, D.H,, Lindsay, J. A. and Loeffler, A. (2015). Genomic insights into the rapid emergence and evolution of MDR in Staphylococcus pseudintermedius. Journal of Antimicrobial Chemotherapy , 70, 997-1007.

Moodley, A., Damborg, P. and Nielsen, S.S. (2014). Antimicrobial resistance in methicillin susceptible and methicillin resistant Staphylococcus pseudintermedius, of canine origin: Literature review from 1980 to 2013. Veterinary Microbiology , 171, 337-341.

Nie, L.B., Zou, Y., Hou, J.L., Liang, Q.L., Cong, W. and Zhu, X.Q. (2019). First report of Neospora caninum seroprevalence in farmed raccoon dogs in China. Acta Tropica, 190, 80-82

Rashid, A. and Kravitz, G.. (2015). Skin and soft tissue infections. Clinical Infectious Diseases , 60, 172-172.

Richards, A.C., O'Shea, M., Beard, P.M., Goncheva, M.I., Tuffs, S.W., Fitzgerald, J.R. and Lengeling, A. (2018). Staphylococcus pseudintermedius Surface Protein L (SpsL) Is Required for Abscess Formation in a Murine Cutaneous Infection Model. Infection and immunity, 86, e00631-18

Ruscher, C., Antina, L.B., Semmler, T., Wleklinski, C.G., Paasch, A., Šoba, A., Stamm, I., Kopp, P., Wieler, L.H. and Walther, B. (2010). Widespread rapid emergence of a distinct methicillin- and multidrugresistant Staphylococcus pseudintermedius (MRSP) genetic lineage in Europe. Veterinary Microbiology , $144,340-346$.

Sasaki, T., Kikuchi, K., Tanaka, Y., Takahashi, N., Kamata, S. and Hiramatsu, K.. (2007). Reclassification of phenotypically identified staphylococcus intermedius strains. Journal of Clinical Microbiology, 45 , 27702778 .

Savini, V., Barbarini, D., Polakowska, K., Gherardi, G., Bialecka, A., Kasprowicz, A., Polilli, E., Marrollo, R., Di Bonaventura. G., Fazii, P., D’Antonio, D., Miedzobrodzki, J. and Carretto, E. (2013). Methicillinresistant Staphylococcus pseudintermedius in a bone marrow transplant recipient. J Clin Microbiol , 51, 1636.

Shao, X.Q., Wen, Y.J., Ba, H.X., Zhang, X.T., Yue, Z.G., Wang, K.J., Li, C.Y., Qiu, J. and Yang, F.H. (2014). Novel amdoparvovirus infecting farmed raccoon dogs and arctic foxes. Emerging Infectious Diseases, $20,2085-2088$.

Silva, M.B., Ferreira, F.A., Garcia, L.N., Silva-Carvalho, M.C., Botelho, L.A., Figueiredo, A.M. and Vieirada-Motta, O. (2015). An evaluation of matrix-assisted laser desorption ionization time-of-flight mass spectrometry for the identification of Staphylococcus pseudintermedius isolates from canine infections. $J$ Vet Diagn Invest, 27, 231-235. 
Somayaji, R., Priyanth, M.A.R., Rubin, J.E. and Church, D. (2016a) Human infections due to Staphylococcus pseudintermedius, an emerging zoonosis of canine origin: report of 24 cases. Diagnostic Microbiology and Infectious Disease , 85, 471-476

Somayaji, R., Rubin, J.E., Priyantha, M. and Church, D. (2016b). Exploring staphylococcus pseudintermedius: an emerging zoonotic pathogen. Future Microbiology, 11, 1371-1374

Song, Y., Yu, L., Zhang, Y., Dai, Y., Wang, P., Feng, C., Liu, M., Sun, S., Xie, Z. and Wang, F. (2020). Prevalence and characteristics of multidrug-resistant mcr-1-positive Escherichia coli isolates from broiler chickens in Tai'an, China. Poult Sci, 99, 1117-1123.

Stegmann, R.B.A., Maranta, C.A. and Perreten, V. (2010). Human infection associated with methicillinresistant Staphylococcus pseudintermedius ST71. J Antimicrob Chemother , 65, 2047-2048

Sun, J., Dang, L.X., Liu, G.S., Cao, Y.S. and Sun, J.H. (2012). Cloning and Sequence Analysis of MC1R Gene in Reddish-brown Wusuli Raccon Dog.Chinese Agricultural Science Bulletin , 28, 41-48 (In Chinese).

van Duijkeren, E., Catry, B., Greko, C., Moreno, M.A., Pomba, M.C., Pyoraia, S., Ruzauskas, M., Sanders, P., Threlfall, E.J., Torren-Edo, J. and Tärneke, K. (2011). Review on methicillin-resistant Staphylococcus pseudintermedius. J Antimicrob Chemother , 66, 2705-2714.

Van Hoovels, L., Vankeerberghen, A., Boel, A., Van Vaerenbergh, K. and De Beenhouwer, H. (2006). First case of Staphylococcus pseudintermedius infection in a human. Journal of clinical microbiology, 44, 46094612 .

Wang, C., Zhu, Y., Li, F. and Huang, L. (2017). The Effect of Lactobacillus isolates on growth performance, immune response, intestinal bacterial community composition of growing Rex Rabbits. Journal of Animal Physiology 83 Animal Nutrition, 101, e1-e13

Wang, L.H., Tang, Q. and Liang, G.D. (2014). Rabies and rabies virus in wildlife in mainland China, 19902013. International Journal of Infectious Diseases , 25, 122-129

Windahl, U., Reimegärd, E., Holst, B.S., Egenvall, A., Fernström, L., Fredriksson, M., Trowald-Wigh, G. and Andersson, U.G. (2012). Carriage of methicillin-resistant Staphylococcus pseudintermediusin dogs-a longitudinal study. BMC Veterinary Research , 8, 34-40.

Wioletta, K. and Maria, S.E. (2018). Are zoonotic staphylococcus pseudintermedius strains a growing threat for humans?. Folia Microbiologica . DOI:10.1007/s12223-018-0615-2

Xu, C., Ma, X.S., Zhang, H., Zhang, X.X., Zhao, J.P., Ba, H.X., Xiu, R.D., Xing, M., Wang, Q.K. and Zhao, Q. (2016). Prevalence, risk factors and molecular characterization of Enterocytozoon bieneusi in raccoon dogs (Nyctereutes procyonoides ) in five provinces of Northern China. Acta Tropica, 161, 68-72.

Yu, J.Y., Zhu, Q., Diao, F.F., Teng, C.J., Peng, H., Shang Y.Y., Zhao,Y.F., Wang, J.L., Jiang, S.J. and Xie, Z.J. (2018). Emergence of novel canine parvovirus type 2 and its pathogenesis in raccoon dogs. Veterinary Microbiology , 216, 7-12.

\section{Hosted file}

Figures . doc available at https://authorea.com/users/309889/articles/440751-emergence-of-a-noveldisease-caused-by-staphylococcus-pseudintermedius-in-raccoon-dogs-nyctereutes-procyonoides

\section{Hosted file}

Tables . doc available at https ://authorea. com/users/309889/articles/440751-emergence-of-a-noveldisease-caused-by-staphylococcus-pseudintermedius-in-raccoon-dogs-nyctereutes-procyonoides 